\title{
Navigation Systems Requirements for Airborne Interferometric SAR Platforms
}

\author{
Marc BARA, Jordi MONNÉ, Antoni BROQUETAS \\ Universitat Politècnica de Catalunya (UPC). Campus Nord, C/ Jordi Girona, 1-3, 08034 Barcelona, Spain. \\ Phone / Fax: +34 93401 1065/+3493 401 7232. E-mail: mabara@voltor.upc.es
}

\begin{abstract}
It is well known that by means of airborne interferometric SAR systems it is possible to generate high precision Digital Elevation Models (DEM), although these platforms are seriously affected by motion instabilities. These deviations are recorded by navigation systems, such as GPS receivers and inertial units. The precision of these systems, and the subsequent off-line processing, determines the level of confidence in the generated heights. Errors in track knowledge lead to incorrect generation of the interferometric phase, which yields height errors in the final DEM. In this paper it is shown an analytical work which is useful to analyze the consequences of such factors onto the bias and the standard deviation of the height errors in Digital Elevation Models.
\end{abstract}

\section{INTRODUCTION}

The unstable movements of an airborne SAR platform can be corrected during the processing step [1], as long as the aircraft deviations are recorded in an accurate manner. Indeed, motion compensation methods are based on the exact knowledge of both antenna positions for each transmitted pulse. These positions are usually obtained after a differential GPS off-line processing, integrated with inertial data, in order to achieve the maximum accuracy.

Any remaining position error will not be compensated during SAR processing and, therefore, will introduce degradation in the final interferometric product. It is worth commenting that we will divide the problem in several parts: in the first section we will consider constant position errors along the synthetic aperture, while in the following section linear-terms (velocity) in the three axes will be taken into account. Then, a discussion on possible effects of errors in attitude angles will be presented.

Finally, it is also interesting to establish the in-flight requirements of maximum deviations from the nominal track, since they determine the performance of the on-board navigation systems.

\section{POSITION ACCURACY}

In a single-pass interferometric system, a position error affects both tracks in the same way. This means that important parameters like baseline are not affected, as long as rotations of the aircraft are well recorded. Therefore, the position errors in trajectories are translated directly to the final DEM as a constant height and ground mismatch. With the use of only one ground control point this error could be avoided.

The situation is more critical in a repeat-pass configuration, since the inaccurate position is different in both tracks. Thus, the baseline itself is affected, yielding important geometric errors during the geocoding step. Equation (1) shows the basic equations used to obtain the DEM, which are based on the antenna position $P_{i}$, the distance to the target $r_{i}$, and its position $P_{0}$, where $i=1,2$. The errors are modelled with the random variable $\Delta P_{i}$, which is considered as a gaussian with a zero mean and a specific standard deviation.

$$
\left|\overline{P_{i}}+\overline{\Delta P_{i}}-\overline{P_{0}}\right|=r_{i}
$$

The problem appears when $\Delta P$ takes different values in both tracks, like in a repeat-pass acquisition. Since the error has zero mean, the final map accuracy will be also characterised by a zero mean error in a statistical sense, but it will present a certain degree of height and ground error depending on the specific error of the flight. To assess this effect, a simulation with the parameters of the DLR E-SAR system configured in this mode [2] with a horizontal baseline of $50 \mathrm{~m}$ has been carried out. The results are shown in Fig. 1, where the error standard deviation in height is displayed along the swath as a function of aircraft position accuracy, considering that the result is corrected with a central control point.

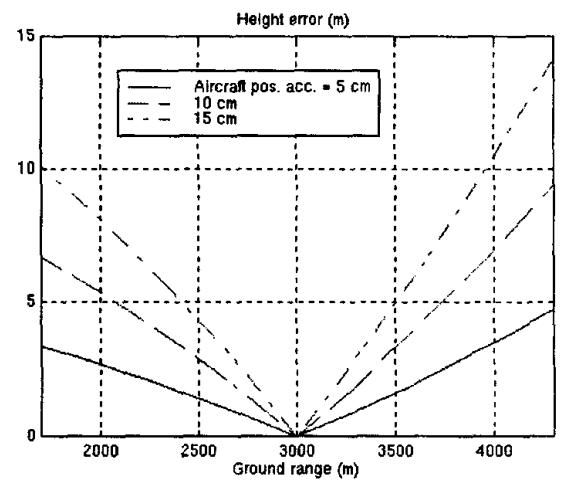

Figure 1. Height error for a repeat-pass system

As we can see, precisions in track registration of $5 \mathrm{~cm}$ are needed to limit the errors below $5 \mathrm{~m}$. For this value, the ground mismatch on the swath edge is around $2 \mathrm{~m}$. One conclusion is that this kind of airborne SAR configuration imposes strong requirements to the differential correction and off-line processing of GPS and inertial data. 


\section{VELOCITY MISMATCH}

For the processing of the raw data, it is necessary to know the forward speed of the platform during the data acquisition. Any mismatch between the real speed and the estimated one will cause a Doppler rate error and, consequently, distortions on the impulse response of a target [3]. It can be shown that after the azimuth compression stage, the remaining phase error can be expressed as a series expansion around the Doppler centroid frequency $f_{c}$. In this way, the more general case of squinted geometry is taken into account:

$$
\phi_{\text {error }}=\frac{4 \pi}{\lambda} r\left\{\begin{array}{l}
\cos (\beta) \cdot\left(1-\sqrt{1-\frac{2 \Delta v}{v_{a}} \tan ^{2}(\beta)}\right)+ \\
+\left(\frac{\lambda}{2 v_{a}}\right)^{2} \frac{f_{c}}{\cos (\beta)} \cdot \frac{2 \Delta v}{v_{a}+2 \Delta v}\left(f_{a}-f_{c}\right)+ \\
+\left(\frac{\lambda}{2 v_{a}}\right)^{2} \frac{1}{\cos ^{3}(\beta)} \frac{\Delta v}{v_{a}+2 \Delta v}\left(f_{a}-f_{c}\right)^{2}
\end{array}\right\}
$$

where $\lambda$ is the wavelength, $r$ is the zero-Doppler distance, $f_{a}$ the azimuth frequency, $\boldsymbol{\beta}$ the squint angle, $v_{a}$ the velocity assumed by the processor, and $\Delta v$ is the difference with respect to the real speed $v$. The interferometric phase error is easily found by subtracting the error in each channel.

It is worth remarking that the azimuth shift induced by the linear term is different in each channel, as it depends on the range distance. Thus, in the case of a perfect coregistration of both signal peaks, there would be an additional phase error. It would be caused by this misregistration, in combination with the azimuth phase ramp coming from the Doppler centroid. In fact, each target is not affected by this additional phase bias at the original azimuth position.

In order to validate (2), a real data set from the DLR E-SAR system [4] has been used. The scene imaged is the test site over Oberpfaffenhofen, with five corner reflectors used as reference ground control points (GCP). The raw data has been processed with a squint angle of $2.7 \mathrm{deg}$ and velocity errors ranging from 0 to $15 \mathrm{~cm} / \mathrm{s}$. With these parameters, the interferometric phase error is found to be negligible, as predicted by (2); but not the azimuth shift in each channel, as shown in Fig. 2 for the near-range target. Table 1 shows the measured and expected displacements of each reflector for errors of 5 and $15 \mathrm{~cm} / \mathrm{s}$, respectively. As we can see, the measured shift agrees with the theoretical one predicted by (2). It is worth realizing that this shift only appears in the squinted case.

Table 1. Shift results $(\mathrm{m})$ with real data

\begin{tabular}{lcccc}
\hline & $\Delta \nu=5 \mathrm{~cm} / \mathrm{s}$ & \multicolumn{3}{c}{$\Delta v=15 \mathrm{~cm} / \mathrm{s}$} \\
\hline & Expected & Measured & Expected & Measured \\
P0 & 0.24 & 0.23 & 0.73 & 0.70 \\
P1 & 0.25 & 0.23 & 0.75 & 0.70 \\
P2 & 0.26 & 0.26 & 0.77 & 0.70 \\
P3 & 0.26 & 0.26 & 0.78 & 0.71 \\
P4 & 0.27 & 0.22 & 0.80 & 0.72 \\
\hline
\end{tabular}

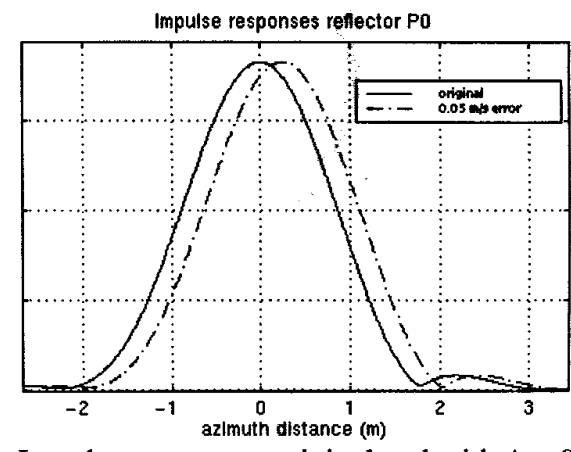

Figure 2. Impulse responses, original and with $\Delta v=0.05 \mathrm{~m} / \mathrm{s}$

Another possible source of error comes from imprecisions in the perpendicular velocity. This factor can cause unknown linear displacements along the synthetic aperture, which yield distortions after compressing the SAR image. These errors can be also developed in a series expansion, with similar consequences as in (2). To be specific, it can be demonstrated that there exists an azimuth shift of $\Delta t \mathrm{~s}$ of the response peak, and a zero-order phase error $\Delta \phi_{0}$ given by:

$$
\begin{gathered}
\Delta \phi_{0}=\frac{2 \pi}{\lambda} \frac{r}{\cos \beta}\left(\frac{v_{p}}{v}\right)^{2} \\
\Delta t=\frac{r}{\cos ^{2} \beta} \frac{v_{p}}{v^{2}}
\end{gathered}
$$

where $v_{p}$ is the perpendicular velocity on the slant-range plane. The expression of $\Delta t$ is similar to that shown in [5] with the inclusion of the squint angle. Indeed, it represents the main limitation in the velocity measurement. For instance, for a typical forward velocity of $90 \mathrm{~m} / \mathrm{s}$, a slant range distance of $5 \mathrm{~km}$, and azimuth resolution of $0.5 \mathrm{~m}, v_{p}$ should be on the order of $1 \mathrm{~cm} / \mathrm{s}$ to limit the shift below a resolution cell. For this value, the phase error of (3) would be negligible. As a conclusion of this section, it is possible to state that velocity measurements must be carried out in an accurate way, mainly in the perpendicular axes. Equations (2) and (3) are a useful tool to evaluate these requirements in each case.

\section{ATTITUDE ANGLES}

The most critical attitude angle to be measured by the navigation units is the roll angle, since it determines directly the orientation of the baseline in a single-pass system. Any imprecision $\Delta \alpha$ in roll imposes a bias $\Delta h$ in the determination of the height, given by:

$$
\Delta h=\Delta \alpha \cdot r \cdot \sin \theta
$$

where $\theta$ is the incidence angle. This error leads to systematic slopes in range direction, as well as topographydependent imprecisions all over the imaged scene. This effect has been checked with the E-SAR data (Fig. 3). A roll error of $0.05 \mathrm{deg}$ has been simulated during geocoding, resulting in a linear evolution of the height error from 2.5 to $4 \mathrm{~m}$, as we can see in Fig. 4 (right). Moreover, inaccuracies of around $7 \mathrm{~cm}$ 
have been detected in buildings with a height of $10 \mathrm{~m}$ (left). In order to limit these distortions below $1 \mathrm{~m}$, the gyroscopes would need accuracies of around $0.01 \mathrm{deg}$. Otherwise, the use of GCP is required to improve the baseline knowledge.

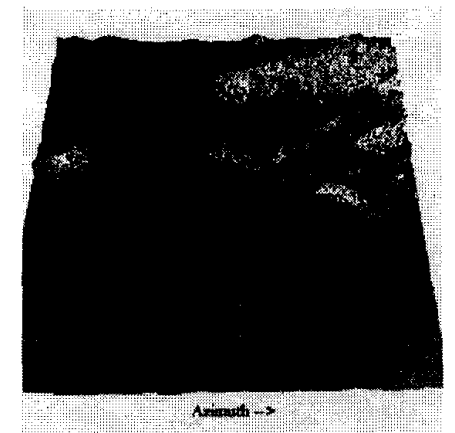

Figure 3. DEM with height errors
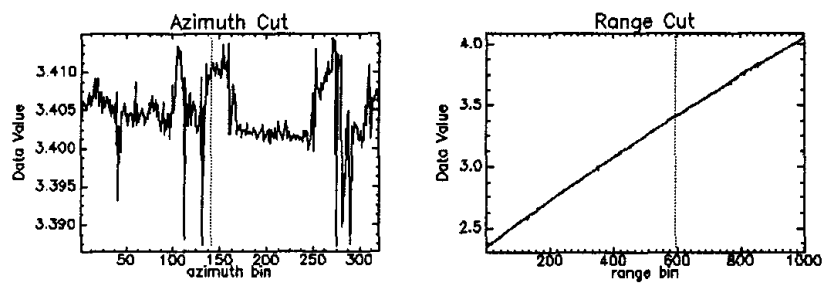

Figure 4. Height errors in range and azimuth $(\Delta \alpha=0.05 \mathrm{deg})$

\section{IN-FLIGHT REQUIREMENTS}

Even if the aircraft trajectory is perfectly known, the fact that motion compensation methods must correct deviations from the nominal path can introduce a certain degree of error in the interferometric phase, as described in [6]. This implies that the SAR platform should follow the nominal straight track as accurate as possible, what imposes the sensitivity limits to the in-flight performance of navigation systems.

Basically, motion compensation induces errors coming from the formation of the synthetic aperture and the unknown topography. In the first case, reference [4] studies the problem considering that range-dependent motion compensation is applied before the range migration (RM) correction, what yields a phase error when combining both channels. If motion compensation were applied accurately to the RM-corrected response (using for instance the two-step scheme described in [1]) this type of error would be avoided. However, for long apertures, the fact that the target position in azimuth is not known means that a residual error is introduced on the edges of the aperture, as a result of the difference of viewing angle to the center. For instance, considering a constant displacement $\Delta d$ to be corrected along the track, there would exist a slight error of the Doppler rate. It can be shown that it leads to a phase error in the peak response given by:

$$
\Delta \phi \approx \frac{\pi \lambda \cdot \cos \beta \cdot \Delta d}{24 \cdot \delta_{x}{ }^{2}}
$$

where $\delta_{x}$ is the azimuth resolution. In an interferometric system, the phase error would be the subtraction of (5) for both channels. Then, as the baseline increases the $\Delta d$ factor is different in the two responses, producing a higher error. However, for typical parameters of a single-pass system (for example in X-Band, baseline $=1.5 \mathrm{~m}$, resolution $=0.5 \mathrm{~m}$ ) a horizontal displacement of the platform equal to $50 \mathrm{~m}$ only produces a phase shift of $0.01^{\circ}$, which is totally negligible. In a repeat-pass system the displacement would be different in both responses and (5) should be taken into account.

The main source of limitation comes from the second type or error, which appears due to the unknown topography during motion compensation. As an example, for a terrain height of $500 \mathrm{~m}$ and a compensated displacement of $20 \mathrm{~m}$, the interferometric phase error reaches the value of $10^{\circ}$. Roll variations are more harmful since they imply different displacement in both antennas. For the same case, a roll fluctuation of $1^{\circ}$ produces a phase bias of $15^{\circ}$. In order to avoid these phenomenons, it would be recommendable that navigation units provide the necessary knowledge to maintain a nominal flight track on the order of $+/-5 \mathrm{~m}$ and $+/-1 \mathrm{deg}$ in roll for a typical low-height airborne case.

\section{CONCLUSIONS}

Airborne SAR interferometric sensors demand a precise knowledge of the track during the data acquisition, otherwise the final elevation models degrade. In this paper we have derived the theoretical expressions which compute the degree of error coming from possible inaccuracies of the on-board navigation systems. This study is useful for determining the sensitivity thresholds that such units should fulfil for highquality mapping applications.

\section{ACKNOWLEDGMENT}

The authors would like to thank the German Aerospace Establishment (DLR) for providing the data used in this work.

\section{REFERENCES}

[1] A. Moreira, J. Mittermayer, R. Scheiber, "Extended Chirp Scaling Algorithm for Air- and Spaceborne SAR Data Processing in Stripmap and ScanSAR Imaging Modes", IEEE TGRS, September 1996.

[2] A. Ulbricht, A. Reigber, "Airborne Repeat-Pass Interferometry with DLR's Experimental SAR (ESAR): First Results", EUSAR'98.

[3] R. Bamler, D. Just, "Phase Statistics and Decorrelation in SAR Interferograms", IGARSS'93.

[4] R. Horn, "The DLR Airborne SAR Project E-SAR", IGARSS'96.

[5] D. Blacknell et al., "Geometric Accuracy in Airborne SAR Images”, IEEE TAES, March 1989.

[6] D. Stevens, I. Cumming, A. Gray, "Options for Airborne Interferometric SAR Motion Compensation", IEEE TGRS, March 1995. 